\title{
Presence of Abnormal Amounts of Dolichols in the Urinary Sediment of Batten Disease Patients
}

\author{
N. M. K. NG YING KIN AND LEONHARD S. WOLFE(23) \\ Department of Neurochemistry, Montreal Neurological Institute and Hospital, McGill University, \\ Montreal, Canada H3A $2 B 4$
}

\begin{abstract}
Summary
Polyisoprenols of the dolichol class have been identified in urinary sediment for the first time and quantified by a high performance liquid chromatographic method. In the late infantile and juvenile forms of Neuronal Ceroid Lipofuscinosis (Batten disease), greatly increased amounts of dolichols of C-90 to C-105 were found in the urinary sediment compared with a variety of other neurologic disorders and age-matched normal subjects. Dolichols purified from the urinary sediment of a late infantile Batten disease patient were shown to have spectroscopic and chemical properties identical to standard preparations of liver and brain dolichols.
\end{abstract}

\section{Speculation}

Measurement of dolichols in the urinary sediment could be of value in diagnosis and screening of siblings of Batten disease. The discovery that dolichols are present in the storage cytosomes, are increased in both the brain and dehisced renal tubular cells in the urine suggests that the basic biochemical defect in these inherited disorders lies on pathways involved in the utilization of dolichols for glycoprotein synthesis.

The group of familial cerebromacular degenerations of childhood termed neuronal ceroid lipofuscinosis by Zeman et al. (21) consist of several phenotypes based on age of onset and clinical course. The late infantile and juvenile forms are often referred to as Batten disease. A pathologic hallmark of these conditions is the intracellular accumulation of abnormal cytosomes filled with materials that exhibit a strong greenish-yellow autofluorescence in both neuronal and non-neuronal tissues. Electron microscopy of the storage material shows characteristic ultrastructural patterns described as curvilinear and fingerprint profiles (4). Electron microscopy of urinary sediment from the late infantile cases of Batten disease also shows the presence of curvilinear bodies (5), which are derived from the renal tubular epithelium (6).

The storage material in neurones of the cerebral cortex has been isolated previously in highly purified form and subjected to chemical analyses $(10,17)$. The insoluble autofluorescent residue remaining after repeated extractions with chloroform-methanol mixtures was tentatively identified as containing retinoid-like complexes to protein (17). The extracted lipids consisted mainly of unesterified cholesterol and phospholipids. More recently, the presence of significant amounts of dolichols has been identified in the storage material by high performance liquid chromatography $(19,20)$.

We describe here the isolation and characterization of unesterified dolichols in the urinary sediment of late infantile and juvenile cases of Batten disease. Dolichols were also measured in the urinary sediment of normal subjects and other neurologic conditions and compared with the Batten disease group.

\section{MATERIALS AND METHODS}

Pig liver dolichols (Sigma Chemical Co., St Louis, MO) were used as standards in high performance liquid chromatography (HPLC) and thin layer chromatography (TLC) analyses. TLC was performed on Eastman Kodak silica gel chromatogram sheets using toluene as the developing solvent. The compounds were visualized first with iodine vapour and then with the anisaldehyde spray reagent of Dunphy et al. (9). Reversed-phase HPLC was performed on a Waters Associates system (Waters Associates, Inc., Mississauga, Ontario) equipped with a Model 450 Variable Wavelength UV Detector operated at $210 \mathrm{~nm}$. Ten $\mu \mathrm{m}$ RP-8 (Brownlee Labs., Santa Clara, CA) $25 \times 0.4 \mathrm{~cm}$ column or a $\mu$ Bondapak $\mathrm{C}_{18}$ (Waters Associates) $30 \times 0.4 \mathrm{~cm}$ column was used with methanolisopropanol $65: 35$ or 50:50 as elution solvent systems at a flowrate of $2 \mathrm{ml} / \mathrm{min}$.

Dolichols were isolated from fresh or fresh-frozen 24-h urine samples as follows: $300 \mathrm{ml}$ of the urine samples were centrifuged at $4000 \mathrm{Xg}$ for $30 \mathrm{~min}$ at $4^{\circ} \mathrm{C}$. The sediments were then extracted with 20 volume chloroform-methanol (2:1 by volume), filtered, and subjected to Folch washings. The lower phase contents were then dried and weighed. Dolichols were extracted with $3 \times 1.5 \mathrm{ml}$ boiling hexane. After removal of the solvent, the residue was redissolved in $0.5 \mathrm{ml}$ of hot hexane, allowed to cool overnight at $4^{\circ} \mathrm{C}$, filtered, and evaporated to dryness. The residue was then dissolved in $100 \mu$ l hexane and $10 \mu \mathrm{l}$ injected into the HPLC system for estimation of the dolichols. The total amount of unesterified dolichols and dolichol fatty acid esters were determined by subjecting the hexane extracts to mild alkaline hydrolysis in $0.5 \mathrm{M}$ potassium hydroxide in $95 \%$ ethanol for $2 \mathrm{~h}$ at $90^{\circ} \mathrm{C}$ before HPLC determination of the dolichols. For determination of dolichol phosphoryl compounds, the residue remaining after hexane extraction was subjected to strong alkaline hydrolysis as described by Breckenridge and coworkers (3) before HPLC analyses. The areas under the HPLC peaks corresponding to C-90-C-105 dolichols were integrated and compared to standard amounts of pure pig liver dolichols.

Approximately $50 \mu \mathrm{g}$ amounts of dolichols were purified from a liter of urine from a Batten disease patient by silica gel open column chromatography and HPLC for Fourier transform proton magnetic resonance (F.T.p.m.r.) spectroscopy on a Brucker WH400 spectrometer. The compound was dissolved in $300 \mu \mathrm{l}$ of deuterochloroform $\left(\mathrm{CDCl}_{3}\right)$. The lock signal was on the deuterium nucleus and the chemical shifts $(\delta)$ were in ppm relative to tetramethylsilane. An aliquot of the purified dolichols from urine was converted to the acetate derivative by reacting with acetic anhydride/pyridine at $80^{\circ} \mathrm{C}$ for ( 1$) \mathrm{h}$ for comparison by TLC and HPLC with authentic dolichol acetates.

\section{RESULTS}

A typical HPLC elution profile for the dolichols from an aliquot of a 24-h urine collection from a patient with the late infantile form of Batten disease is shown in Figure 1. Using liver dolichol 


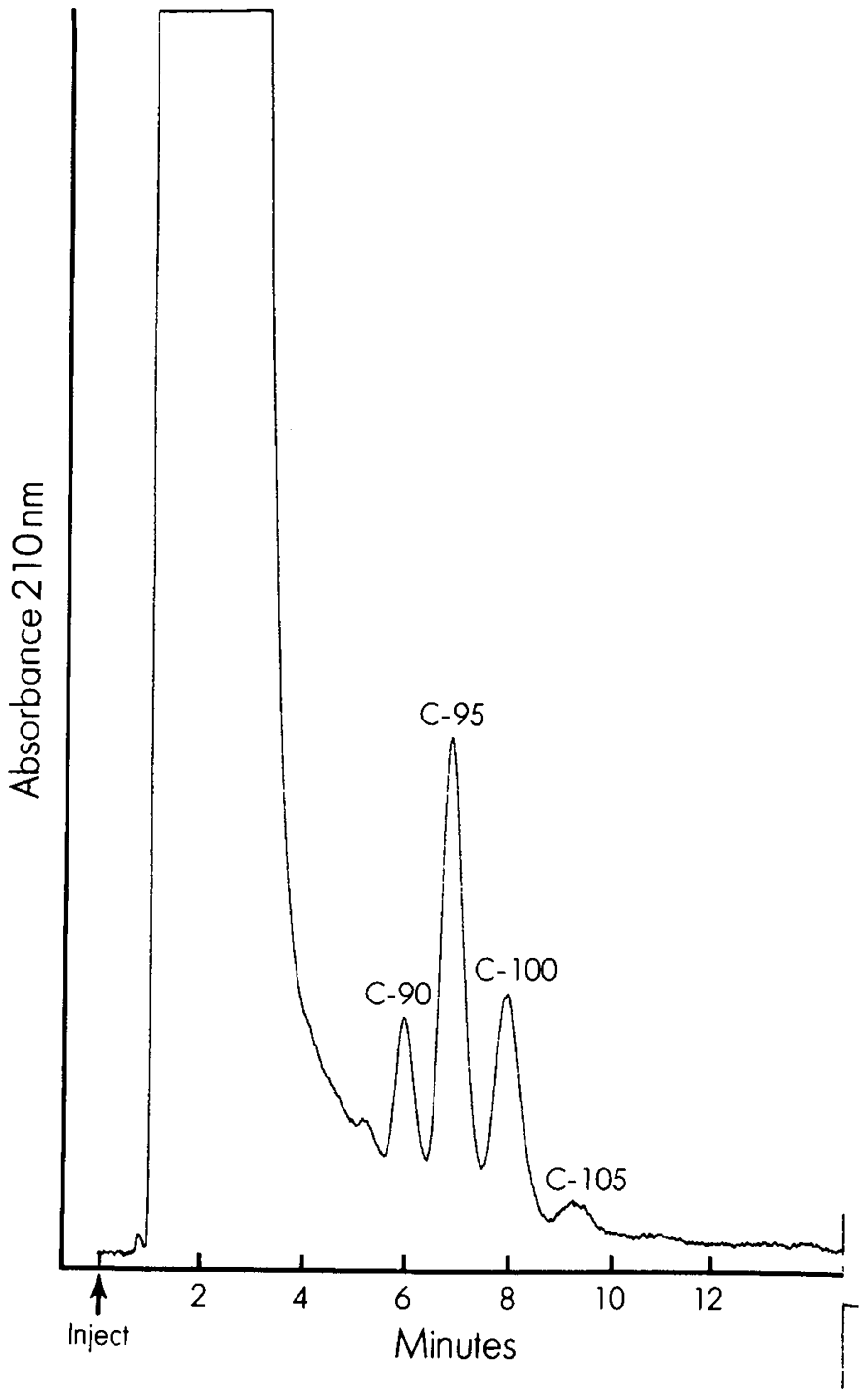

Fig. 1. Example of high performance liquid chromatographic separation of dolichols from the urinary sediment of a patient diagnosed as having the late infantile form of Batten disease. The numbers above the peaks refer to the carbon chain length of the dolichols.

as standards, the peaks eluting between 5-10 min were identified as the C-90-C-105 homologues. From the peak area measurements, the amount of dolichols excreted per mg of total lipid was calculated to be $7.4 \mu \mathrm{g}$ for this patient as compared to less than 0.5 $\mu \mathrm{g}$ for non-Batten disease patients. Measurement of the dolichols excreted in the urinary sediment of Batten disease patients, together with other neurologic disorders and storage diseases as well as normal subjects is shown in Table 1 . It is clear that Batten disease patients excreted at least ten times the amount of dolichols than other patients and age-matched normal subjects.

For more definitive identification of the dolichols excreted by Batten disease patients, lipids from the sediments of several 24- $h$ urine collections from a patient in the Montreal area were purified as described in the "Methods and Material" section. The free alcohols from the urine when subjected to TLC comigrated as a single spot $\left(R_{F} 0.3\right)$ with reference liver dolichols and also gave the characteristic yellow-green colour when developed with the anisaldehyde reagent. After treatment with the acetylating reagents, the resulting dolichol acetates from both the urine sample and the liver standards again comigrated as a single spot but with an $R_{F}$ of 0.7 indicating the less polar nature of the acetylated products. The acetate derivatives by HPLC analysis were found
Table 1. Dolichols in urinary sediment from Bátten disease and other subjects

\begin{tabular}{|c|c|}
\hline Diagnosis & $\begin{array}{c}\mu \mathrm{g} \text { dolichols } / \mathrm{mg} \\
\text { total lipid }\end{array}$ \\
\hline \multicolumn{2}{|c|}{ Late infantile Batten disease (Jansky- } \\
\hline \multicolumn{2}{|c|}{ Bielschowsky type) } \\
\hline \multicolumn{2}{|l|}{ Patient 1 (R. B., age 9) } \\
\hline Collection 1 & 4.1 \\
\hline Collection 2 & 8.2 \\
\hline Collection 3 & 3.0 \\
\hline Collection 4 & 7.0 \\
\hline Patient 2 (J. R., age 3) & 7.4 \\
\hline Mean \pm S.D. & $5.94 \pm 2.26$ \\
\hline \multicolumn{2}{|c|}{ Juvenile Batten disease (Spielmeyer-Vogt type) } \\
\hline \multicolumn{2}{|l|}{ age $9-20$} \\
\hline Patient 1 (K. P.) & 5.2 \\
\hline Patient 2 (G. G.) & 4.2 \\
\hline \multicolumn{2}{|l|}{ Patient 3 (M. A.) } \\
\hline Collection 1 & 6.4 \\
\hline Collection 2 & 3.5 \\
\hline Patient 4 (M. M.) & 3.3 \\
\hline Mean \pm S.D. & $4.52 \pm 1.29$ \\
\hline \multicolumn{2}{|l|}{ Other Neurological diseases } \\
\hline Niemann-Pick, Type C & 0.17 \\
\hline Sialidosis, normosomatic type & 0.00 \\
\hline Tay Sachs & 0.18 \\
\hline \multicolumn{2}{|l|}{ Epilepsy patients (ages 9-30) } \\
\hline Collection 1 & 0.03 \\
\hline Collection 2 & 0.11 \\
\hline Collection 3 & 0.07 \\
\hline Collection 4 & 0.18 \\
\hline Collection 5 & 0.13 \\
\hline Collection 6 & 0.54 \\
\hline \multicolumn{2}{|l|}{ Friedreichs ataxia } \\
\hline Collection 1 & 0.21 \\
\hline Collection 2 & 0.07 \\
\hline Alzheimers disease & 0.21 \\
\hline Chromosomal abnormality & 0.00 \\
\hline Mean \pm S.D. & $0.146 \pm 0.14$ \\
\hline \multicolumn{2}{|l|}{ Normal subjects (ages 10-18) } \\
\hline Collection 1 & 0.03 \\
\hline Collection 2 & 0.03 \\
\hline Collection 3 & 0.00 \\
\hline Collection 4 & 0.30 \\
\hline Collection 5 & 0.10 \\
\hline Collection 6 & 0.00 \\
\hline Collection 7 & 0.20 \\
\hline Mean \pm S.D. & $0.094 \pm 0.12$ \\
\hline
\end{tabular}

to have not only the same elution patterns as the underivatised dolichols, but also the same elution volumes when compared to one another; however, the acetate derivatives were eluted later.

The F.T.p.m.r. spectrum of $50 \mu \mathrm{g}$ of the major dolichols isolated from the late infantile Batten disease urine was superimposable with spectra of purified calf brain dolichols or liver dolichols (3) with typical major absorptions of the repeating isoprenoid units in dolichols, $\delta 5.14(\mathrm{C}=\mathrm{C}-H), \delta 2.06\left(\mathrm{C}=\mathrm{C}-\mathrm{CH}_{2}\right)$, and $\delta 1.70$ $\left(\mathrm{C}=\mathrm{C}-\mathrm{CH}_{3}\right)$.

Hexane extracts from urinary sediments of Batten disease and other subjects before and after alkaline hydrolysis yielded the same amount of dolichols. HPLC analyses of the untreated hexane extracts using methanol:isopropanol (35:65) also showed no fatty acid esters. Further, when the residue remaining after exhaustive hexane extraction of the chloroform: methanol fraction was subjected to strong alkaline hydrolysis, no dolichol could be detected, the hexane extracts containing most of the dolichols. It would thus appear that the dolichols present in the urinary sediments are unesterified. 


\section{DISCUSSION}

The histochemical and the electron microscopic appearance of the storage material in biopsy specimens is at present a key component in the diagnosis of children suspected of having one or other forms of the neuronal ceroid lipofuscinoses. There has been much controversy on the chemical nature of the storage material and the basic biochemical defect in these recessively inherited storage disorders is at present unknown. On the basis of the detailed studies on the autofluorescent products produced during lipid peroxidation in biologic systems (8), Zeman (22) postulated that the ceroid-lipofuscin storage derived from peroxidation products of unsaturated fatty acids. Armstrong and coworkers $(1,2)$ indeed reported deficiencies in leukocyte peroxidase in Batten disease patients; however, other investigators including our own group failed to demonstrate such a deficiency (12-16, 19).

Recent studies in our laboratory on the chemical nature of the storage materials in Batten disease have shown that they consisted of a chloroform-methanol soluble fraction and an autofluorescent residue in approximately equal proportions. The insoluble materials were shown to have properties similar to retinoid complexes (17-19). In the lipid soluble solvent extracts, polyisoprenols of the dolichol class were also identified $(19,20)$. No evidence of products of lipid peroxidation were found. More recently, we have shown that dolichols are present in four to ten times larger amounts in the cerebral cortex of Batten disease compared with age-matched non-Batten disease patients (20, and unpublished results). De Baecque (5) has suggested that analysis of urinary sediments by electron microscopy offers a convenient noninvasive method for the diagnosis of Batten disease. In this paper, we clearly show an excessive excretion of dolichols in the urine of both late infantile and juvenile types of these diseases. These compounds most probably originate from sloughed renal tubular cells. Thus, the measurement of dolichols in urinary sediment of patients can provide, for the first time, a biochemical indicator of these diseases and may prove helpful in screening of siblings in families with a child already affected.

The storage of retinoid-like compounds and dolichols in Batten disease patients suggests a possible defect in the recycling of dolichol and retinyl compounds during the biosynthesis of glycoproteins because both retinol and dolichol are known lipid intermediates in this process $(7,11)$. Alternatively, if the primary defect in Batten disease is in the metabolism of retinyl compounds, as we suggested earlier, the accumulated retinoid complexes could interfere with pathways involved in the synthesis of dolichyl phosphates.

\section{REFERENCES AND NOTES}

1. Armstrong, D., Dimmit, S., and Van Wormer, D. E.: Studies in Batten disease. I. Peroxidase deficiency in granulocytes. Arch. Neurol., 30: 144 (1974).

2. Armstrong, D., Dimmit, S., Boehme, D. H., Leonberg, S. C., and Vogel, W.: Leucocyte peroxidase deficiency in a family with a dominant form of Kufs' disease. Science, 186: 155 (1974).

3. Breckenridge, W. C., Wolfe, L. S., and Ng Ying Kin, N. M. K.: The structure of brain polyisoprenols. J. Neurochem., 2I: 1311 (1973).

4. Carpenter, S., Karpati, G., Andermann, F., Jacob, J. C., and Andermann, E. The ultrastructural characteristics of the abnormal cytosomes in Batten-Kufs' disease. Brain, 100: 137 (1977).
5. De Baecque, C.: Diagnosis of neuronal ceroid liopfuscinosis by electron microscopy of urinary sediment. N. Engl. J. Med., 292: 1408 (1975).

6. De Baecque, C., Pollack, A. M., and Suzuki, K.: Systemic pathology in late infantile neuronal storage disease with curvilinear bodies. Arch. Pathol. Lab. Med., 100: 139 (1976).

7. De Luca, L. M.: Vitamin A. In: Handbook of Lipid Research, Vol. 2, The FatSoluble Vitamins, (De Luca, H. F., ed.). pp 1-54 (Plenum Press, New York, 1978).

8. Dillard, C. J. and Tappel, A. L.: Fluorescent products of lipid peroxidation of mitochondria and microsomes. Lipids, 6: 715 (1971).

9. Dunphy, P. J., Kerr, J. D., Pennock, J. F., Whittle, K. J., and Feeney, J.: The plurality of long-chain isoprenoid alcohols (polyprenols) from natural sources. Biochim. Biophys. Acta, 136: 136 (1967)

10. Palo, J., Haltia, M., Wolfe, L. S., and Ng Ying Kin, N. M. K.: Isolation of storage material from the brains of patients with various types of neuronal ceroidlipofuscinosis. 7th. Meeting of the International Society for Neurochemistry, Jerusalem, Sept. 2-6,i, 1979. Abstracts, p. 520.

11. Parodi, A. J. and Leloir, L. F.: The role of lipid intermediates in the glycosylation of proteins in the eucaryotic cell. Biochim. Biophys. Acta, 559: 1 (1979).

12. Pilz, H. and Goebel, H. H.: Peroxidase activity in parotid gland in juvenile neuronal ceroid-lipofuscinosis. Neuropath. Appl. Neurobiol., 3: 93 (1972).

13. Pilz, H., O'Brien, J. S., and Heipertz, R.: Human saliva peroxidase: microanalytical isoelectric fractionation and properties in normal persons and in cases with neuronal ceroid lipofuscinosis. Clin. Biochem., 9: 85 (1976).

14. Pilz, H., Schwendlemann, G., and Goebel, H. H.: Diagnostic significance of myeloperoxidase assay in neuronal ceroid-lipofuscinosis (Batten-Vogt syndrome). Neurology, 28: 924 (1978).

15. Schwerer, B. and Bernheimer, H.: Leucocytes PPD-peroxidase activity with polyunsaturated fatty acid hydroperoxides: normal values in Batten's disease. J. Neurochem., 31: 457 (1978).

16. Sumi, S. M. and Farrell, D. F.: Electron microscopy of skin biopsy and leucocyte peroxidase assay in the diagnosis of juvenile neuronal ceroid lipofuscinosis. Neurology, 26: 364 (1976).

17. Wolfe, L. S., Ng Ying Kin, N. M. K., Baker, R. R., Carpenter, S., and Andermann, F.: Identification of retinoyl complexes $s$ the autofluorescent component of the neuronal storage material in Batten disease. Science, 195: 1360 (1977).

18. Wolfe, L. S.: Biochemical expression of degenerative disorders associated with cognitive dysfunction. IN: Congenital and Acquired Disorders (R. Katzman, Ed.) p 125 (Raven Press, New York, 1979).

19. Wolfe, L. S., Ng Ying Kin, N. M. K., and Baker, R. R.: Batten disease and related disorders: new findings on the chemistry of the storage material. In Lysosomes and Lysosomal Storage Diseases, (J. W. Callahan and J. A. Lowden, Eds.), p 315-330 (Raven Press, New York, 1981).

20. Wolfe, L. S. and Ng Ying Kin, N. M. K.: Dolichols in storage cytosomes and urine sediment of Batten disease cases. Trans. Amer. Soc. Neurochem. (Abstract), 12: 194 (1981).

21. Zeman, W., Donahue, S., Dyken, P., and Green, J.: The neuronal ceroidlipofuscinosis (Batten-Vogt Syndrome). In: Handbook of Clinical Neurology, (P. J. Vinken and G. W. Bruyn, Eds.) Vol 10 p 588 . Elsevier, Amsterdam, 1970).

22. Zeman, W.: Presidential addres: studies in the neuronal ceroid-lipofuscinosis. J. Neuropath. Exp. Neurol., 33: 1 (1974).

23. Requests for reprints should be addressed to: Dr. L. S. Wolfe, Montreal Neurologicl Institute, 3801 University Street, Montreal, Que., Canada H3A 2B4.

24. We wish to thank the following physicians for their help in obtaining 24-h urine samples from their patients: Dr. Frederick Andermann, Dr. Serge Gauthier and Dr. Allan Sherwin of the Montreal Neurological Institute; Dr. Gordon Watters, Montreal Children's Hospital; Dr. Bernard Lemieux, Centre Hospitalier, Sherbrooke, Que.; Dr. Gail Solomon, New York Hospital-Cornell Medical Center, New York; Dr. Michael Blaw, The University of Texas Health Science Center at Dallas, Texas; Dr. Sherman, Valley Children's Hospital and Guidance Clinic, Fresno, California; and Dr. Burton Waisbren, Milwaukee Children's Hospital, Milwaukee, Wisconsin. We also thank Ms Pat Steele for technical help and Dr. H. Beierbeek of the Laboratoire régional de RMN à haut champ. Université de Montréal, Montréal, Québec for the measurement of the F.T.p.m.r. spectra. This research was supported by Medical Research Council of Canada grant MT-1345.

25. Received for publication September 4, 1981.

26. Accepted for publication January 8, 1982. 